\title{
The Stability of Spontaneous and Ultraviolet-Induced Reversions from Auxotrophy in Escherichia coli
}

\author{
By RUTH F. HILL \\ Radiological Research Laboratory, Department of Radiology, \\ Columbia University, New York, U.S.A.
}

(Received 1 June 1962)

\section{SUMMARY}

Spontaneous and u.v.-induced revertants were found to be unstable in serial subculture. Instability was manifested by $(a)$ replacement of the original revertant by revertants with slower growth rates on minimal medium, and $(b)$ the appearance of auxotrophic organisms which overgrew in some cases but not others. In one case, the instability was found to be more pronounced when the organisms were maintained in the logarithmic phase of growth. This evidence, along with the previous radiological evidence, was interpreted according to the following hypothesis. The auxotrophic organism has an episomic element located at the suppressor locus. The damage inflicted by radiation consists in the detachment of this episome. The prototrophic cell may again become auxotrophic by reattachment.

\section{INTRODUCTION}

Previous investigation (Hill, 1962) of the u.v. dose-mutation relationship for the reversion of Escherichia coli strain WP2 from tryptophan-dependence to independence suggested that the observed revertants might not have been produced at random in the population of irradiated auxotrophic organisms but rather might have been produced in a select fraction. This fraction would consist of organisms which had a greater degree of stationary phase metabolism before irradiation and, associated with it, a greater resistance to the lethal effect of u.v.-irradiation. The logical extension of this concept leads to the possibility that if the unirradiated population of auxotrophic organisms was uniform and had the maximum possible degree of stationary phase metabolism before irradiation, every surviving cell would be prototrophic. It seemed to us that this conclusion implied something unique about the mutation from auxotrophy to prototrophy in WP2. To determine whether this might be true, a study of the revertants was made. The results are reported here.

\section{METHODS}

Bacterial strains. The revertants used in these experiments were derived from the tryptophan-requiring strains, Escherichia coli WP2 and WWP2, described previously (Hill, 1962). Spontaneous and u.v.-induced revertants were used.

Culture conditions. Cultures of the revertants were maintained by serial transfer in nutrient broth. One-tenth $\mathrm{ml}$. of each culture was diluted into $2 \mathrm{ml}$. of broth for transfer and the dilution incubated without aeration at $37^{\circ}$ overnight. This allowed 
about 4-5 divisions to occur in each subculture. In one set of experiments, to be described in more detail later, transfer was also made by diluting the overnight culture into $10 \mathrm{ml}$. broth and growing with aeration at $37^{\circ}$. When a culture was to be studied for its content of organisms, after dilution into fresh broth, the remainder of the culture was centrifuged, re-suspended in physiological saline solution, diluted in saline and 200-300 organisms plated in duplicate on MM and on SEM agar. As described previously, $\mathbf{M M}$ is minimal medium $\mathbf{E}$ solidified with washed agar and SEM is the same but supplemented with $5 \%$ broth. In experiments where the requirement of secondary auxotrophs arising among the prototrophs was to be determined, MM was supplemented with $40 \mu \mathrm{g}$. tryptophan $/ \mathrm{ml}$. or with $20 \mu \mathrm{g}$. phenylalanine or tyrosine. These last two amino acids were used to determine whether the secondary auxotrophs were less specific in their requirement than the primary auxotrophs WP2 and WWP2. In all cases, the secondary auxotrophs were found to require tryptophan specifically.

Isolation of revertants. Spontaneous and u.v.-induced revertant colonies were obtained by plating saline suspensions of unirradiated and irradiated WP2 and WWP 2 on SEM or on MM. To be reasonably certain that the mutant colonies appearing when irradiated cultures were plated were not of spontaneous origin, the volume plated on SEM was chosen to give about 100 mutant colonies. The background of spontaneous mutant colonies, arising during the growth of the auxotrophic parent on SEM was usually 6-15. On MM this precaution was unnecessary since the parent strains cannot grow and the frequency of spontaneous mutants capable of forming colonies on MM did not exceed about one per $10^{8}$ organisms. A very small portion of each revertant colony was carefully scraped from the surface with a needle and suspended in $1 \mathrm{ml}$. of saline. Several dilutions of this suspension were placed in duplicate on SEM and on MM so as to observe the initial characteristics of the revertant. These plates were incubated at $37^{\circ}$ and examined daily to determine the rate of growth on MM and any changes that might appear on SEM. After 3 days of incubation, scrapings from one or two colonies were put into $2 \mathrm{ml}$. broth. The original saline suspension was also diluted as $0.1 \mathrm{ml}$. into $2 \mathrm{ml}$. of broth. Both sets of broth cultures-one set inoculated from the revertant colony when it first appeared and the other set inoculated with a subclone derived from it-were then maintained by serial subculture (the cultures were refrigerated between subcultures). After every few subcultures, the cultures were studied as described above.

Terminology. Various authors have reported that revertants arising among amino acid-dependent organisms vary in their ability to grow on unsupplemented minimal medium (Smith-Keary, 1958; Stadler \& Yanofsky, 1959; Strauss \& Okubo, 1960; Bacon \& Treffers, 1961). The revertants are usually classified according to the time required for the colony size on MM to reach the size produced by the wild type after $24 \mathrm{hr}$. of incubation at $37^{\circ}$. Thus they may be $\mathrm{F}$ (fast) meaning that the growth rate is indistinguishable from that of the wild-type; $S 48$, (slow, $48 \mathrm{hr}$. required); S72, etc. The same terminology is used in the present study.

\section{RESULTS}

Fourteen independent spontaneous revertants and 18 subclones derived from them were studied first. Of the 14, four were fast types when first examined and remained fast. This was also characteristic of the subclones derived from them. 
The rest of the 10 original cultures and the 15 subclones derived from them were of the S48 type initially. However, after various numbers of subcultures, all of these 25 began to show signs of instability. The instability was manifested in the following ways:

(a) Gradual overgrowth by a cell type which gave a colony on SEM with the same morphology as that produced by WP2 (small, grey and translucent) and which did not form a colony on MM. Whenever this colony type appeared, the colony was tested and invariably found to be tryptophan-dependent (Pl. 1, fig. 1).

(b) Marked variation in the sizes of the colonies on MM (Pl. 1, fig. 2, 3). In some cases, this feature preceded overgrowth by auxotrophic organisms; in others, it persisted for some time and then disappeared, only to appear again at a later time. In the latter cases, an occasional or a few auxotrophic colonies appeared but the culture was not overgrown. A detailed study of one such case will be presented later.

(c) In some cases, as the percentage of $\mathrm{S} 48$ types decreased, they were replaced not only by auxotrophic organisms but also by a very much slower type of revertant which did not produce a visible pinpoint-size colony on MM until after 3-5 days of incubation (Pl. 1, fig. 4). Sometimes the culture became stabilized for many subcultures with a mixture of auxotrophs and very very slow revertants. Eventually the very very slow type declined in favour of the auxotrophic type.

Although the four cultures and their subclones which were classified as fast remained fast, an occasional slight variation in colony size on MM was observed. Also an auxotrophic colony appeared occasionally.

An interesting finding was that cultures of subclones did not always show exactly the same pattern of instability as the colony from which they were isolated. For example, in one case, the original culture became a mixture of $10 \%$ auxotrophic cells and $90 \%$ very very slow revertants by the twenty-first subculture. It then remained stable at this ratio through the next nineteen subcultures. On the other hand, cultures of two subclones from this parent were overgrown by auxotrophic cells by the 9 th and 11 th subcultures. In one, the overgrowth seemed to be gradual and complete, i.e. no revertants were found when $10^{6}$ organisms were plated; in the other, a ratio of $1 \% \mathrm{~S} 48$ revertants and $99 \%$ auxotrophs was maintained through six subcultures.

Revertants induced by u.v.-irradiation were studied next. After u.v.-irradiation of WP2 and WWP2, more than $95 \%$ of the revertant colonies were slow in appearing when plating was done on MM. Bacon \& Treffers (1961) reported that, in the case of spontaneous reversion from ornithine-dependence, the growth rate of the colony, as it first appeared on MM, did not indicate its characteristic rate. Colonies which did not reach full size until $96 \mathrm{hr}$. of incubation initially, often turned out to be fast types after subculture. However, this was not observed with irradiated WP2 and WWP2. Colonies which were slow in appearing initially were also slow after subculture.

The series of u.v.-induced revertants used for study included equal numbers of S 48 and S72 types. A small fraction of types appearing even more slowly on SEM and MM were found, but these were not used. Ten of the revertants were obtained from WP2 and eight from WWP2. No difference due to the auxotrophic parent was observed. Both the S48 and S72 types and subclones derived from them 
exhibited the same patterns of instability as the spontaneous revertants. Instability did not appear to depend on whether the revertant was $\mathbf{S} 48$ or S72.

One u.v.-induced revertant was studied in some detail. This mutant was originally S48. It began to show variations in colony size on MM by the 19th subculture. By the 34th subculture, this variation was still observed (PI. 1, fig. 2) and in addition a small percentage of the colonies appearing on SEM were tryptophan-dependent. When the smaller-sized colonies on MM were picked and re-plated, they turned out to be S72 and S96 types. The 34th subculture was done on 17 August 1961. In spite of bi-weekly subculture by $1 / 20$ dilution in broth since then, there has been no change in the percentage of auxotrophic organisms and the colony size variation on MM has persisted until the time of writing-April 1962. However, a way to change this picture was discovered.

In September 1961, the following experiment was performed. It was repeated twice in February 1962, with some modifications of procedure but essentially the same results. The experiment consisted simply of $(a)$ growing by aeration in broth, (b) sampling frequently, and (c) comparing the properties of the organisms when the culture was allowed to complete one growth cycle with the properties when the culture was maintained in the logarithmic phase. Details of the experiment and the results are shown in Tables 1 and 2.

\section{Table 1. Instability of u.v.-induced revertant no. 103 as a function of the growth cycle}

An overnight broth culture grown without aeration was diluted 1/1000 into fresh broth and aerated at $37^{\circ}$. Two $\mathrm{ml}$. samples were removed every $2 \mathrm{hr}$., the organisms washed in saline, diluted and plated. After $8 \mathrm{hr}$, , the culture was refrigerated overnight. The next day, it was diluted $1 / 4000$ and the experiment repeated.

\begin{tabular}{|c|c|c|c|c|c|c|}
\hline \multirow{3}{*}{$\begin{array}{l}\text { Time of } \\
\text { sampling } \\
\text { (hr.) }\end{array}$} & \multicolumn{4}{|c|}{$\begin{array}{l}\text { Organisms } / \mathrm{ml} \text {. calculated from nos. of colonies appearing } \\
\text { after } 48 \mathrm{hr} \text {. incubation of plates containing }\end{array}$} & \multirow{2}{*}{\multicolumn{2}{|c|}{$\begin{array}{l}\text { Nos. of cols. on } \mathrm{MM} \times 100 \text {, } \\
\text { Nos. of prototroph cols. on } \\
\text { SEM when MM plates } \\
\text { were incubated for }\end{array}$}} \\
\hline & \multirow{2}{*}{$\begin{array}{l}\mathrm{MM}+40 \mu \mathrm{g} . \\
\text { tryptophan } \\
\text { per ml. }\end{array}$} & \multicolumn{3}{|c|}{ SEM } & & \\
\hline & & Total & $\%$ proto. & $\%$ auxo. & $48 \mathrm{hr}$. & $72 \mathrm{hr}$. \\
\hline o & $2.9 \times 10^{5}$ & $2.6 \times 10^{5}$ & 96.7 & $\mathbf{3} \cdot \mathbf{3}$ & 100 & 100 \\
\hline 2 & $5.9 \times 10^{6}$ & $6.3 \times 10^{6}$ & $97 \cdot 0$ & $\mathbf{3 \cdot 0}$ & 54 & 89 \\
\hline 4 & $1.1 \times 10^{8}$ & $1.0 \times 10^{7}$ & $95 \cdot 6$ & $4 \cdot 4$ & 84 & 100 \\
\hline 6 & $6.1 \times 10^{8}$ & $5.6 \times 10^{8}$ & $92 \cdot 5$ & $7 \cdot 5$ & 85 & 104 \\
\hline 8 & $1.1 \times 10^{9}$ & $9.2 \times 10^{8}$ & $91 \cdot 6$ & $8 \cdot 4$ & 92 & 100 \\
\hline 0 & $3.0 \times 10^{5}$ & $2.9 \times 10^{5}$ & $95 \cdot 5$ & $4 \cdot 5$ & 72 & 88 \\
\hline 2 & $2.6 \times 10^{6}$ & $2.5 \times 10^{6}$ & $96 \cdot 0$ & 4.0 & 75 & 88 \\
\hline 4 & $4 \cdot 1 \times 10^{7}$ & $4 \cdot \Theta \times 10^{7}$ & $91 \cdot 0$ & $9 \cdot 0$ & 63 & 71 \\
\hline 6 & $3.9 \times 10^{8}$ & $3.2 \times 10^{8}$ & $88 \cdot 4$ & $11 \cdot 6$ & 65 & 92 \\
\hline 8 & $1.7 \times 10^{9}$ & $1.6 \times 10^{9}$ & $84 \cdot 7$ & $15 \cdot 3$ & 83 & 100 \\
\hline 24 & $2.3 \times 10^{9}$ & $2.0 \times 10^{9}$ & 80.0 & $20 \cdot 0$ & 100 & 100 \\
\hline
\end{tabular}

At the beginning of the experiment, the culture contained about $3 \%$ of auxotrophic organisms. Although there was a variation in the size of the colonies formed on MM, all of the prototrophic organisms produced colonies on this medium which were visible after $48 \mathrm{hr}$. of incubation. As shown in Table 1, during the logarithmic phase of the growth cycle, the percentage of auxotrophic organisms increased slightly. More importantly, the colonies formed by many of the prototrophic organisms on MM took longer to appear. Both of these effects became more pronounced when the culture 
was put through a second cycle of growth. When the culture entered the stationary phase, the rate of growth of the prototrophic organisms on MM increased again. Refrigeration of the culture when in the late logarithmic stage of growth seemed to cause a decrease in both the percentage of auxotrophic organisms and the rate of growth of prototrophic organisms on MM but this phenomenon was not investigated.

\title{
Table 2. Instability of u.v.-induced revertant no. 103 when kept in the logarithmic growth phase
}

\begin{abstract}
These experiments were performed simultaneously with those described in Table 1 and the same overnight culture was used initially. This was diluted 1/100 and aerated. After $2 \mathrm{hr}$., a 1/100 dilution was made; the dilution was then aerated another $2 \mathrm{hr}$; the remainder of the original culture was sampled in the same manner as the accompanying culture (see Table 1). This procedure was repeated every $2 \mathrm{hr}$. The dilution made after a total time of $8 \mathrm{hr}$. was allowed to aerate overnight. The next day, this was sampled, diluted 1/1000 initially and the procedure of the previous day repeated. The 1/1000 dilution was also sampled.
\end{abstract}

\begin{tabular}{|c|c|c|c|c|c|c|}
\hline \multirow{3}{*}{$\begin{array}{l}\text { Time of } \\
\text { dilution } \\
\text { (hr.) }\end{array}$} & \multicolumn{4}{|c|}{$\begin{array}{l}\text { Organisms } / \mathrm{ml} \text {. calculated from nos. of colonies appearing } \\
\text { after } 48 \mathrm{hr} \text {. incubation of plates containing }\end{array}$} & \multirow{2}{*}{\multicolumn{2}{|c|}{$\begin{array}{l}\text { Nos. of cols. on } \mathrm{MM} \times 100 \\
\begin{array}{c}\text { Nos. of proto. cols.on SEM } \\
\text { when MM plates were } \\
\text { incubated for }\end{array}\end{array}$}} \\
\hline & \multirow{2}{*}{$\begin{array}{l}\mathrm{MM}+40 \mu \mathrm{g} \\
\text { tryptophan } \\
\text { per ml. }\end{array}$} & \multicolumn{3}{|c|}{ SEM } & & \\
\hline & & Total & $\%$ proto. & $\%$ auxo. & $48 \mathrm{hr}$. & $72 \mathrm{hr}$ \\
\hline 0 & $3.0 \times 10^{8}$ & $3.0 \times 10^{8}$ & $96 \cdot 7$ & $\mathbf{3 \cdot 3}$ & 95 & 100 \\
\hline 2 & $56 \times 10^{7}$ & $4.2 \times 10^{7}$ & $96 \cdot 0$ & $4 \cdot 0$ & 91 & 100 \\
\hline 4 & $1.1 \times 10^{7}$ & $1.1 \times 10^{7}$ & 93.5 & $6 \cdot 5$ & 76 & 88 \\
\hline 6 & $2 \cdot 3 \times 10^{6}$ & $2 \cdot 1 \times 10^{6}$ & $91 \cdot 4$ & $8 \cdot 6$ & 88 & 95 \\
\hline 8 & $3.4 \times 10^{5}$ & $3.2 \times 10^{5}$ & 86.5 & $13 \cdot 5$ & 79 & 90 \\
\hline 24 & $3.4 \times 10^{9}$ & $3.1 \times 10^{9}$ & $69 \cdot 8$ & $30 \cdot 2$ & 47 & 70 \\
\hline $\mathbf{2 4}$ & $3.5 \times 10^{6}$ & $3.4 \times 10^{6}$ & $70 \cdot 9$ & $29 \cdot 1$ & $\mathbf{8 8}$ & 77 \\
\hline 26 & $4.3 \times 10^{7}$ & $4.6 \times 10^{7}$ & $70 \cdot 8$ & $29 \cdot 2$ & 34 & 57 \\
\hline 28 & $1.0 \times 10^{7}$ & $1.3 \times 10^{7}$ & $52 \cdot 8$ & $47 \cdot 2$ & 18 & 41 \\
\hline 30 & $3.0 \times 10^{6}$ & $3.9 \times 10^{6}$ & $49 \cdot 4$ & $50 \cdot 6$ & 12 & 48 \\
\hline 32 & $8.3 \times 10^{5}$ & $9.0 \times 10^{5}$ & $38 \cdot 0$ & $62 \cdot 0$ & 19 & 67 \\
\hline
\end{tabular}

Table 2 shows the results of keeping the culture in the logarithmic phase for an extended time. There is a more pronounced decrease in the rate of growth of the prototrophic organisms on MM and a more rapid increase in the fraction of auxotrophic organisms.

\section{DISCUSSION}

It was suggested previously (Hill, 1962) that the number of u.v.-induced revertants which can be detected is limited by the selective medium, since those requiring higher broth concentrations would be masked by the increased growth of auxotrophic organisms. A second source of limitation is indicated by the presentobservation that, after irradiation of the auxotrophic parent, some revertants do not appear on SEM or MM until after 4 or more days of incubation. If even slower ones were present, they might produce only microcolonies. Still another source of limitation may be the phenomenon of instability. If instability happens to occur within the first few divisions after the newly induced prototroph starts to grow on agar, the prototrophic organisms remaining in such a clone might not produce a visible colony.

The finding of instability as a characteristic of both spontaneous and u.v.-induced 
reversions from tryptophan dependence in Escherichia coli, WP2 and WWP2, is in agreement with observations made on spontaneous leucine-independent revertants in Salmonella typhimurium by Smith-Keary (1958). As in the case of the revertants derived from WP2 and WWP2, the slow types derived from $S$. typhimurium leushowed different degrees of instability manifested as variations in the fraction of auxotrophic organisms and in the sizes of colonies formed by the prototrophic organisms on MM. In addition, revertant clones which appeared initially to be stable slow types frequently became unstable after subculture (Dawson \& Smith-Keary, 1962). Although instability was reported only for the slow types, our results suggest that the faster types are probably unstable also.

The slow types in the Salmonella case were shown by genetic analysis to be due to mutation at a suppressor locus (Smith-Keary, 1960). Although genetic analysis of Escherichia coli B-the wild-type parent of WP2 and WWP2-has not been done, many genetic studies of $E$. coli $\mathrm{K} 12$ have been reported. In this strain, suppressor mutations in tryptophan auxotrophs are common and most of the revertants obtained by u.v.-irradiation are the result of this type of mutation (Stadler \& Yanofsky, 1959). It seems safe to assume that this is true for $E$. coli B also.

Dawson \& Smith-Keary (1962) showed that the most probable explanation for the instability of the leucineless prototrophs is the attachment of an episomic element (Jacob \& Wollman, 1961) to the suppressor locus. Their data also indicate that the episome which attaches to the leucine suppressor locus can also attach to the proline suppressor locus. Furthermore, instability at the leucine suppressor locus was found to be more frequent when this locus was transduced into an arabinose-negative strain, which presumably had more episomes available for attachment than the original leucineless strain. This ubiquity and non-specificity of attachment of this type of episome accounts for the observation that instability may appear at any time. A reasonable hypothesis capable of explaining all of the present findings can be made, if it be assumed that the instability of the tryptophanless revertants studied here, is due similarly to the presence of episomes.

According to Dawson \& Smith-Keary, the attachment of an episome to the mutated suppressor gene causes an increased rate of mutation to auxotrophy. Thus the variations in colony size on MM are due to mutation during the growth of the prototrophic clones on minimal agar. The experiment with the unstable tryptophanless revertant (Tables 1 and 2) shows that the rate of mutation to auxotrophy during growth on minimal agar is increased if the organisms were in the logarithmic phase in broth before transfer to minimal agar. Conversely, when the cells are transferred from the stationary phase, the rate of mutation to auxotrophy on the plate is decreased. This phenomenon is the reverse of what is observed when the mutation to prototrophy from auxotrophy is induced by u.v.-irradiation. In the latter case, the number of revertants appearing on minimal agar increases as the auxotrophic organisms go from the logarithmic to the stationary phase in complete medium, before irradiation and subsequent plating on agar (Hill, 1962). If the change from prototrophy to auxotrophy is due to the attachment of an episome to the suppressor locus, it seems likely that the reverse change from auxotrophy to prototrophy in these strains is due to detachment of an episome from the suppressor locus.

Dawson \& Smith-Keary take the view that the original leucineless strain becomes prototrophic by mutation of the suppressor locus (when the mutation is clearly not 
at the leu- locus) and that the attachment of an episome increases the probability that the mutated suppressor locus will back-mutate to its original inactive state. The present findings suggest that the mutation of the suppressor locus causing prototrophy is the detachment of an episome from this locus and that the backmutation to auxotrophy is the attachment itself rather than something which is caused by the attachment. An interesting corollary of this hypothesis is that the reason why the suppressor locus is ordinarily inactive is that it is masked by having an episome attached to it.

According to our hypothesis, the effect of u.v.-radiation on WP2 and WWP2 is then the same as the effect of radiation on inducible lysogenic strains of bacteria (Lwoff, Siminovitch \& Kjeldgaard, 1950). There are at least three features which are common both to the induction of prototrophy and the change from the prophage to the vegetative state:

(1) There is an optimal dose of u.v. in the low dose-range which gives a maximum effect (Jacob \& Wollman, 1953).

(2) In the case of Escherichia coli $\mathrm{K} 12(\lambda)$, the optimal dose is about 80 sec. for a u.v.-lamp of the same type as the one used in the present investigation and a lamp to sample distance of $80 \mathrm{~cm}$. (Weigle \& Delbrück, 1951). For a lamp to sample distance of $56 \mathrm{~cm}$., which was used by us, the optimal dose would be $40 \mathrm{sec}$. The dose at which the mutagenic effect for the change from auxotrophy to prototrophy seems to be saturated is about $60 \mathrm{sec}$. (Hill, 1962). The agreement in doses, to a first approximation, is to be expected since the radiosensitivities of $E$. coli $\mathrm{K} 12$ and E. coli $\mathrm{B} / \mathrm{r}$ are similar (Zelle, 1955).

(3) Following irradiation, a period of incubation in rich medium is necessary to obtain the maximum yield of induced lysogenic bacteria (Weigle \& Delbrück, 1951) and the maximum yield of revertants. Furthermore, chloramphenicol treatment after irradiation decrease the yield of revertants (Witkin, 1956). According to our hypothesis, a decreased yield means either a failure of the episome to detach from the suppressor locus or immediate re-attachment. Chloramphenicol treatment has been reported to increase the probability that the phage type of episome will be attached (Bertani, 1957; Christensen, 1957).

Cells containing unattached episomes can be 'disinfected' by treating with acridine dyes (Hirota \& Iijima, 1957; Watanabe \& Fukasawa, 1961). This effect is attributed to prevention of multiplication of the episomes while they are cytoplasmic, followed by dilution amongst daughter cells during division. The same dyes increase the yield of u.v.-induced reversions from auxotrophy (Witkin, 1961). If reversion consists of the detachment of an episome, the action of the dyes would be to prevent reattachment. If the revertant is now grown in the presence of the dye, the presumed episome, and any others which are capable of replacing it at the suppressor locus and are cytoplasmic, might be diluted out. The revertant clone would then be stable. Similarly, if the auxotrophic strain is treated with the dye before irradiation, the induced revertants should be stable. Experiments to determine whether these predictions are correct are now in progress.

The removal of an episome as the mechanism responsible for the change to prototrophy in the tryptophanless strains studied here may have general validity for other nutritionally-deficient strains in which suppressor mutations are common. It remains to be seen whether this type of reversion occurring in other strains shows 
the kind of instability reported by Dawson \& Smith-Keary for a leucineless strain and confirmed above for tryptophanless strains. If our hypothesis be correct, then strains in which suppressor mutations have not been detected (Rudner, 1961) may give only stable revertants after irradiation. It would also be interesting to see whether in those strains for which u.v.-radiation is not a mutagen for a particular marker, the spontaneous revertants correspond only to a change at the original locus (Demerec \& Cahn, 1953). In those strains where spontaneous mutations at both the original locus and at a suppressor locus are possible (Stadler \& Yanofsky, 1959) is u.v.-radiation an effective mutagen for both types or only for the mutation at the suppressor locus?

Finally, if the effect of u.v.-irradiation is the detachment of an episome, one may ask, as Lwoff has, whether this may justifiably be called mutation (Lwoff, 1953). Until the discovery of episomic elements, mutation was usually regarded as the result of a stable change in the sequence of bases in DNA. Superficially, the question may seem to be simply a matter of whether one wishes to stretch this definition. However, there is a more important consideration. If it should turn out that u.v.-radiation induces change to prototrophy only by an effect at a suppressor locus and the removal of an episome becomes more than a hypothetical explanation, then the mechanism responsible for other reported types of u.v.-induced mutagenesis would require more extensive investigation. Thus mechanisms other than a change of base in DNA may turn out to be more common than is usually assumed. For example, differential killing and the relief of competitive inhibitory effects are obvious mechanisms by which the mutant fraction of a population would be increased. Unfortunately their possible roles in many reported cases of radiationinduced mutagenesis in bacteria have not been studied.

The author wishes to express gratitude to Dr F. J. Ryan for suggesting the possible presence of an episome, Dr P.F. Smith-Keary for a lively exchange of correspondence and for permission to quote results before publication, and Misses Barbara Potter and Rosanne Levitt for technical assistance. Based on work performed under Contract AT-(30-1)-2740 for the U.S. Atomic Energy Commission.

\section{REFERENCES}

Bacon, D. F. \& Trefrers, H. P. (1961). Spontaneous and mutator-induced reversion of an Escherichia coli auxotroph. I. Prototrophic types and their growth characteristics. J. Bact. 81, 786.

BertaNi, L. E. (1957). The effect of the inhibition of protein synthesis on the establishment of lysogeny. Virology, $4,53$.

Christensen, J. R. (1957). Effect of chloramphenicol on lysogenization by temperate phage P1. Virology, 4, 184 .

Dawson, G. W. P. \& Smith-Keary, P. F. (1962). Episomic control of mutation in Salmonella typhimurium. Heredity, 17 (in the Press).

Demerec, M. \& CahN, E. (1953). Studies of mutability in nutritionally deficient strains of Escherichia coli. J. Bact. 65, 27.

Hru, R. F. (1962). Dose-mutation relationships in ultraviolet-induced reversion from auxotrophy in Escherichia coli. J. gen. Microbiol. 30, 281.

Hrrota, Y. \& IrJma, T. (1957). Acriflavine as an effective agent for eliminating F factor in Escherichia coli K-12. Nature, Lond. 180, 655. 
JACOB, F. \& Wollman, E. L. (1953). Induction of phage development in lysogenic bacteria. Cold Spring Harb. Symp. quant. Biol. 18, 101.

JacoB, F. \& Wollman, E. L. (1961). Sexuality and the Genetics of Bacteria, chap. 16. Academic Press Inc., New York.

Lwoff, A. (1953). Lysogeny. Bact. Rev. 17, 269.

Lwoff, A. Siminovitch, L. \& KJeldgaArd, N. (1950). Induction de la production de bactériophages chez une bactérie lysogène. Ann. Inst. Pasteur, 79, 815.

Rudner, R. (1961). Mutation as an error in base pairing. II. Kinetics of 5-bromodeoxyuridine and 2-aminopurine-induced mutagenesis. Z. Vererb. 92, 361.

Smith-Keary, P. F. (1958). An unstable mutation in Salmonella typhimurium. Nature, Lond. 181, 1672.

Smith-Keary, P. F. (1960). A suppressor of leucineless in Salmonella typhimurium. Heredity, 14, 61.

Stadler, J. \& YaNofSky, C. (1959). Studies on a series of tryptophan-independent strains derived from a tryptophan-requiring mutant of Escherichia coli. Genetics, 44, 105.

Strauss, B. \& OKubo, S. (1960). Protein synthesis and the induction of mutations in Escherichia coli by alkylating agents. J. Bact. 79, 464 .

Watanabe, T. \& Furasawa, T. (1961). Episome-mediated transfer of drug resistance in Enterobacteriaceae. II. Elimination of resistance factors with acridine dyes. J. Bact. $81,679$.

Weigle, J. J. \& Delbrück, M. (1951). Mutual exclusion between an infecting phage and a carried phage. J. Bact. 62, 301.

Wrtkin, E. M. (1956). Time, temperature and protein synthesis: a study of ultravioletinduced mutation in bacteria. Cold Spring Harb. Symp. quant. Biol. 21, 123.

WitKIn, E. M. (1961). Modification of mutagenesis initiated by ultraviolet light through post-treatment of bacteria with basic dyes. J. cell. comp. Physiol. 58, suppl. 1, 135.

ZELLE, M. R. (1955). Radiation induced mutations and their implications on the mechanisms of radiation effects on bacteria. Bact. Rev. 19, 32.

\section{EXPLANATION OF PLATE}

Plate 1. Colony types produced by u.v.-induced revertants after subculture.

Fig. 1. Auxotrophic and revertant colonies formed on SEM by a subclone of no. 101 after 10 subcultures.

Fig. 2. Variations in size of colonies formed on MM by no. 103 after 19 subcultures.

Fig. 3. Variations in size of colonies formed on MM by a subclone of no. 107. This revertant was overgrown by auxotrophic cells by the fifth subculture.

Fig. 4. Colonies of auxotrophs, $S 48$ and $S>96$ revertants formed on SEM by no. 106 after 11 subcultures.

All plates incubated for $48 \mathrm{hr}$ at $37^{\circ}$. 

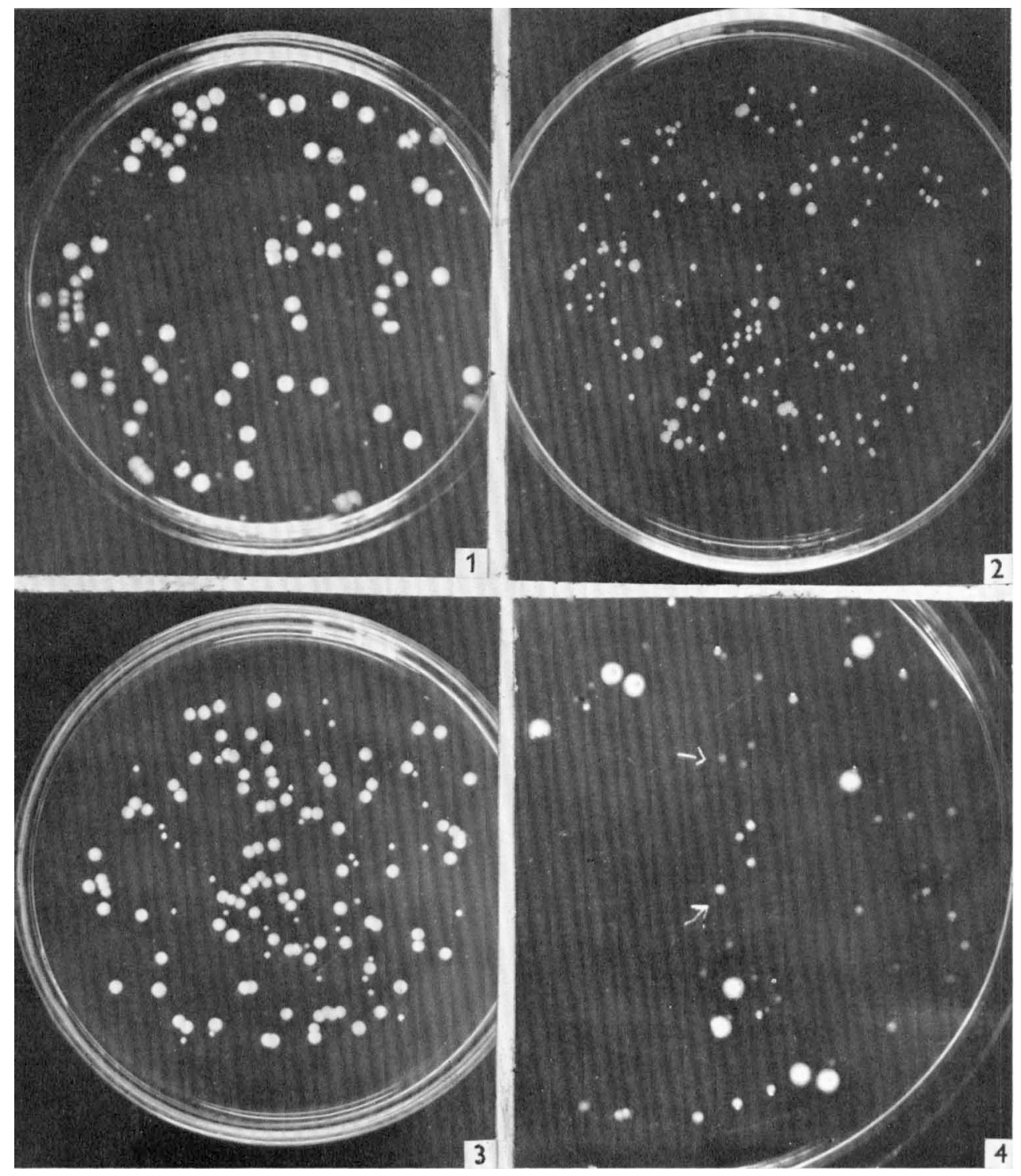

R. F. HILI

(Facing p. 297) 